\title{
Toward a dynamical shift condition for unequal mass black hole binary simulations
}

\author{
Doreen Müller, Bernd Brügmann \\ Theoretical Physics Institute, University of Jena, 07743 Jena, Germany
}

\begin{abstract}
Moving puncture simulations of black hole binaries rely on a specific gauge choice that leads to approximately stationary coordinates near each black hole. Part of the shift condition is a damping parameter, which has to be properly chosen for stable evolutions. However, a constant damping parameter does not account for the difference in mass in unequal mass binaries. We introduce a position dependent shift damping that addresses this problem. Although the coordinates change, the changes in the extracted gravitational waves are small.

PACS numbers: 04.25.D-, 04.25.dg,04.30.Db
\end{abstract}

\section{Introduction}

Early numerical relativity simulations using a $3+1$ split of the Einstein equations suffered from so-called slice stretching, an effect which occurs when using singularity avoiding slicing together with a vanishing shift. The slices become highly distorted when time marches on in the outer regions of the grid but slows down in the vicinity of the black hole. It became clear that a non-vanishing, outwards pointing shift vector would be required in order to redistribute grid points and also to prevent grid points from falling into the black hole. Inspired by Balakrishna et al. [1], Alcubierre et al. [2, 3] combined the $1+\log$ slicing condition with a dynamical shift condition called gamma-driver. These gauge conditions successfully prevented slice stretching in black hole simulations using excision. It turned out that such gauge conditions could be used also for fixed punctures with slight modifications to keep the puncture from evolving [4]. The fixed-puncture modification was removed in [5, 6] when the moving puncture method was introduced. $1+\log$ slicing with gamma-driver shift succeeds in moving the puncture freely through the grid while simultaneously avoiding slice-stretching. The basic reason for the success of this gauge condition is that when the slices start to stretch, the shift vector counteracts by pulling out grid points from the region near the black hole.

In this paper we focus on the dissipation or damping parameter in the gammadriver shift condition, which plays an important role in the success of this gauge. In order to reduce oscillations in the shift vector, the authors of [3] noticed the necessity of a damping term in the shift condition. Adjusting the strength of the damping via a damping parameter was found to allow freezing of the evolution at late times in [4] and to 
avoid drifts in metric variables in [7]. Additionally, the value of the damping coefficient was found to affect the coordinate location of the apparent horizon and therefore the resolution of the black hole on the numerical grid [7, 8]. The right choice of the damping value is therefore important if one wants to resolve the black hole properly while still driving the coordinates to a frame where they are stationary when the physical situation is stationary and hence obtain a stable evolution.

The specific value of the damping parameter has to be adapted to the black hole mass in order to obtain long term stable evolutions. If the damping parameter is either too small or too large, there are unwanted oscillations or a coordinate instability, respectively. In binary simulations, a typical choice is a constant value of roughly $2 / M$, where $M$ is the total mass of the system. However, using a constant damping parameter for black hole binaries with unequal masses leads to a fundamental problem. With a constant damping parameter, the effective damping near each black hole is asymmetric for unequal black hole masses since the damping parameter has dimensions $1 / M$. For large mass ratios, this asymmetry in the grid can be so large that simulations fail because the damping may become too large for one of the black holes. This is one of the reasons why the highest mass ratio that has been successfully simulated up to now is $10: 1[9]$.

Advantageous would be a position-dependent damping parameter that adapts to the local mass, in particular such that in the vicinity of the $i^{\text {th }}$ puncture with mass $M_{i}$ its value approaches $1 / M_{i}$. It was noticed before [4, 9] that a damping coefficient adapted to the various parameters of the simulation would be beneficial. In [10] a position-dependent formula was introduced for head-on collisions of black holes, which to our knowledge was only used in one other publication [11], prior to the moving puncture framework. In this paper, we take first steps towards a position-dependent damping parameter for moving punctures. As a consequence, the local coordinates change compared to standard simulations, but this does not significantly affect gauge invariant quantities like the extracted waves as we discuss below.

\section{Dynamical damping in the shift equation}

\subsection{Numerical setup}

We focus on the gauge condition used in the $3+1$ splitting of the Einstein equations, in particular on the condition for the shift vector.

The slices are determined by the $1+\log$ slicing condition [12] for the lapse function $\alpha$,

$$
\partial_{0} \alpha=-2 \alpha K
$$

where $K$ is the trace of the extrinsic curvature. The coordinates of a given slice are governed by the gamma-driver shift condition introduced in [4] as

$$
\partial_{0}^{2} \beta^{i}=\frac{3}{4} \partial_{0} \tilde{\Gamma}^{i}-\eta_{s} \partial_{0} \beta^{i},
$$


where $\tilde{\Gamma}^{i}$ are the contracted Christoffel symbols of the conformal metric $\tilde{\gamma}_{i j}$, $\beta^{i}$ is the shift vector and $\eta_{s}$ is the damping coefficient we will discuss in this publication. In Eqs. (11) and (2), $\partial_{0}$ is defined as $\partial_{0}=\partial_{t}-\beta^{i} \partial_{i}$ as suggested by [13, 14, 15].

Examining the physical dimensions, we see that $\left[\beta^{i}\right]=1$ and $\left[\partial_{0}\right]=1 / M$, where $M$ is the mass (e.g. the total mass of the spacetime under consideration). For this reason, the second term on the right hand side of equation (2) requires the damping parameter to carry units,

$$
\left[\eta_{s}\right]=\frac{1}{M}
$$

In simulations of a single Schwarzschild puncture of mass $M_{1}$, we typically choose a damping parameter of $\eta_{s} \approx 1 / M_{1}$ for obtaining enough damping in the shift without producing instabilities. In numerical experiments for a Schwarzschild puncture (to be discussed elsewhere), we find that $0 \leq \eta_{s} \lesssim 3.5 / M_{1}$ is necessary for a stable and convergent numerical evolution. Some minimal amount of damping is important to suppress noise in the gauge when a puncture is moving. On the other hand, if $\eta_{s}$ is too large then there are gauge instabilities, leading to a loss of convergence and to instability of the entire numerical evolution. Furthermore, early simulations for fixed punctures also found that $\eta_{s}$ should take values around $1 / M$, where $M$ is the total mass, to avoid long-term coordinate drifts at the outer boundary [4].

In simulations of black hole binaries with total mass $M=M_{1}+M_{2}$, we usually set $\eta_{s}=2 / M$ which has been found to work well in equal mass binaries simulations. (For equal masses, $M_{1}=M_{2}=M / 2$, so near one of the punctures the value of $\eta_{s}$ discussed above for Schwarzschild becomes $\eta_{s}=1 / M_{1}=2 / M$.) For unequal mass binaries, the different black holes tolerate different ranges of $\eta_{s}$ according to the above statement about single black holes. Ideally, $\eta_{s}$ should be $\approx 1 / M_{i}$, which cannot be accomplished simultaneously for unequal masses using a constant value of $\eta_{s}=2 / M$. In fact, for the mass ratio 1:10 in [9], the choice $\eta_{s}=2 / M$ failed, but a smaller value for $\eta_{s}$ was chosen such that $\eta_{s} \lesssim 3.5 / M_{i}$ for both $i=1$ and $i=2$.

To overcome the conflicts between punctures with different masses in evolutions of two or more black holes, we suggest to construct a non-constant, position-dependent damping parameter which knows about the position and mass of each puncture and takes a suitable value at every grid point.

\subsection{Using $\psi^{-2}$ to determine the position of the punctures}

We thus desire a definition of $\eta_{s}$ which respects the unit requirements found in Eq. (3) and which asymptotes to specifiable values at the location of the punctures and at infinity. Typical values are $\eta_{s}=1 / M_{i}$ at the $i^{\text {th }}$ black hole and $\eta_{s}=2 / M$ at large distances. This can be achieved by determining $\eta_{s}$ through a position dependent function defined on the whole grid instead of using a constant as before. We desire a smooth $\eta_{s}$ which avoids modes which travel at superluminal speeds. Since we use the BaumgarteShapiro-Shibata-Nakamura (BSSN) system of Einstein's equations [16, 17], we want 
the form of $\eta_{s}$ to depend only on the BSSN variables in a way that does not change the principal part of the differential operators.

In this paper, we choose to use the conformal factor $\psi$, which contains information about the locations and masses of the punctures. The formula we will use for determining the damping coefficient $\eta_{s}(\vec{r})$ is

$$
\eta_{s}(\vec{r})=\hat{R}_{0} \frac{\sqrt{\tilde{\gamma}^{i j} \partial_{i} \psi^{-2} \partial_{j} \psi^{-2}}}{\left(1-\psi^{-2}\right)^{2}},
$$

with $\tilde{\gamma}^{i j}$ the inverse of the conformal 3-metric and $\hat{R}_{0}$ a dimensionless constant. While $\psi, \tilde{\gamma}^{i j}$, and $\hat{R}_{0}$ are dimensionless, the partial derivative introduces the appropriate dependence on the mass since $\left[\partial_{i}\right]=1 / M$ and hence $\left[\eta_{s}(\vec{r})\right]=1 / M$.

For a single Schwarzschild puncture of mass $M$ located at $r=0$ the behavior of Eq. (4) near the puncture and near infinity is as follows. According to [18], for small radii $r$ (near the puncture) the conformal factor asymptotically equals

$$
\psi^{-2} \simeq p_{1} r
$$

for a known constant $p_{1}$. The next to leading order behavior is less simple [19]. The point $r=0$ corresponds to a sphere with finite areal radius $R_{0}$,

$$
R_{0}=\lim _{r \rightarrow 0} \psi^{2} r=\frac{1}{p_{1}}=\hat{R}_{0} M
$$

Numerically, $\hat{R}_{0} \approx 1.31$. The inverse of the conformal metric behaves like

$$
\tilde{\gamma}^{i j} \simeq \delta^{i j}
$$

Therefore, we find for small $r$

$$
\sqrt{\tilde{\gamma}^{i j} \partial_{i} \psi^{-2} \partial_{j} \psi^{-2}} \simeq p_{1}=\frac{1}{\hat{R}_{0} M}
$$

and

$$
\left(1-\psi^{-2}\right)^{2} \simeq\left(1-p_{1} r\right)^{2} \simeq 1
$$

when keeping only leading order terms in $r$. Equations (8) and (9) combine according to (44) to give

$$
\eta_{s}(r=0)=1 / M
$$

For large $r$ we can expand the conformal factor in powers of $1 / r$,

$$
\psi^{-2} \simeq\left(1+\frac{M}{2 r}\right)^{-2} \simeq 1-\frac{M}{r},
$$

resulting in

$$
\sqrt{\tilde{\gamma}^{i j} \partial_{i} \psi^{-2} \partial_{j} \psi^{-2}}=\frac{M}{r^{2}}
$$

and

$$
\eta_{s}(r \rightarrow \infty) \simeq \hat{R}_{0} \frac{M / r^{2}}{(M / r)^{2}}=\frac{\hat{R}_{0}}{M}
$$


In summary, Eq. (4) leads to

$$
\eta_{s}(r) \rightarrow \begin{cases}1 / M, & r \rightarrow 0 \\ \hat{R}_{0} / M, & r \rightarrow \infty\end{cases}
$$

for a single puncture at $r=0$. Note that using Eq. (41) in Eq. (21) does not affect the principal part of (2). Therefore, the system remains strongly hyperbolic, same as for $\eta_{s}=$ const. according to $[14,13]$.

\section{Results}

Our Eq. (41) analytically gives the desired $1 / M$ behavior near the puncture and near infinity for a single, non-spinning and non-moving puncture. Now it remains to be tested whether these properties persist in actual numerical simulations, especially for unequal mass binaries.

Simulations are performed with the BAM code described in [7, 20]. The code uses the BSSN formulation of Einstein's equations and employs the moving puncture framework [5, 6]. Spatial derivatives are $6^{\text {th }}$ order accurate and time integration is performed using the $4^{\text {th }}$ order Runge-Kutta scheme. The numerical grid is composed of nested boxes with increasing resolution, where the boxes of highest resolution are centered around the black holes. These boxes are advanced in time with Berger-Oligar time stepping [21]. We are using puncture initial data with Bowen-York extrinsic curvature and solve the Hamiltonian constraint using a pseudospectral collocation method described in [22]. The momentum parameter in the Bowen-York extrinsic curvature is chosen such that we obtain quasi-circular orbits in our binary simulations using the method of [23].

For binary simulations with unequal masses, we will use the mass ratio $q=M_{2} / M_{1}$ to denote the runs, $M_{i}$ being the bare mass of the $i^{\text {th }}$ puncture. The physical masses of the punctures (obtained after solving the constraints) differ by less than $10 \%$ from the bare masses for the orbits considered here, so the $\eta_{s}$ values derived for a single puncture should remain valid. When comparing simulations run with $\eta_{s}=2 / M$ and $\eta_{s}(\vec{r})$ following Eq. (4) we will refer to them as "standard" and "new" or "dynamical" gauge, respectively, throughout this paper.

\subsection{Single Schwarzschild Black Hole}

In order to test the $1 / M$-behavior of (4) near the puncture and infinity, we first performed a series of evolutions for a time of $100 \mathrm{M}$ of a single, non-spinning puncture while varying its mass. We then measured the value of $\eta_{s}(\vec{r})$ near the puncture and

at the outer boundary of the grid and compared these values to the limits (14). The data points in Fig. 1 correspond to these measurements while the lines are fits to the numerical data. The values of $\eta_{s}(\vec{r})$ near the puncture as a function of total mass $M$ are fitted to $\eta_{s}(M)=1.05 / M$ which agrees well with the analytical limit $r \rightarrow 0$ of (14). Fitting to $\eta_{s}$ measured near the physical boundary of the grid reveals $\eta_{s}(M)=1.311 / M$ 


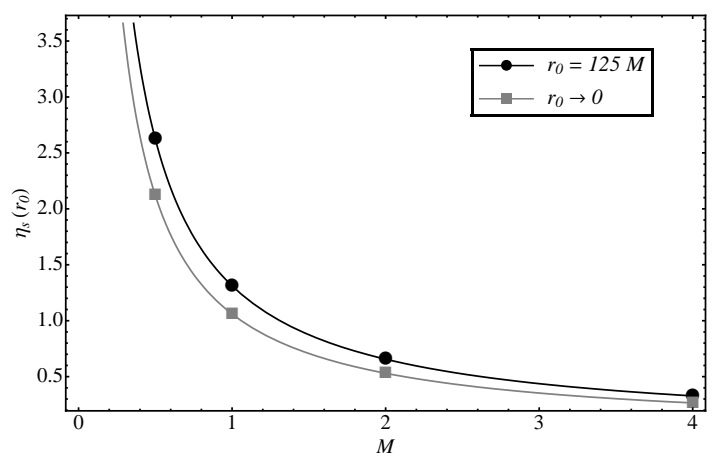

Figure 1. Numerical test of the analytical limits (14) of $\eta_{s}(\vec{r})$ using single, nonspinning punctures with different masses. Shown are the values of $\eta_{s}(r)$ near the puncture (gray squares) and at the outer boundary (black dots). Note that here $M$ is identified with a dimensionless number, so $\eta_{s}(r)$ is dimensionless as well. The fits to the data points are consistent with the analytic prediction. Numerically, $\eta_{s}(M)=1.05 / M$ (gray line) near the puncture and $\eta_{s}(M)=1.311 / M$ (black line) at the outer boundary.
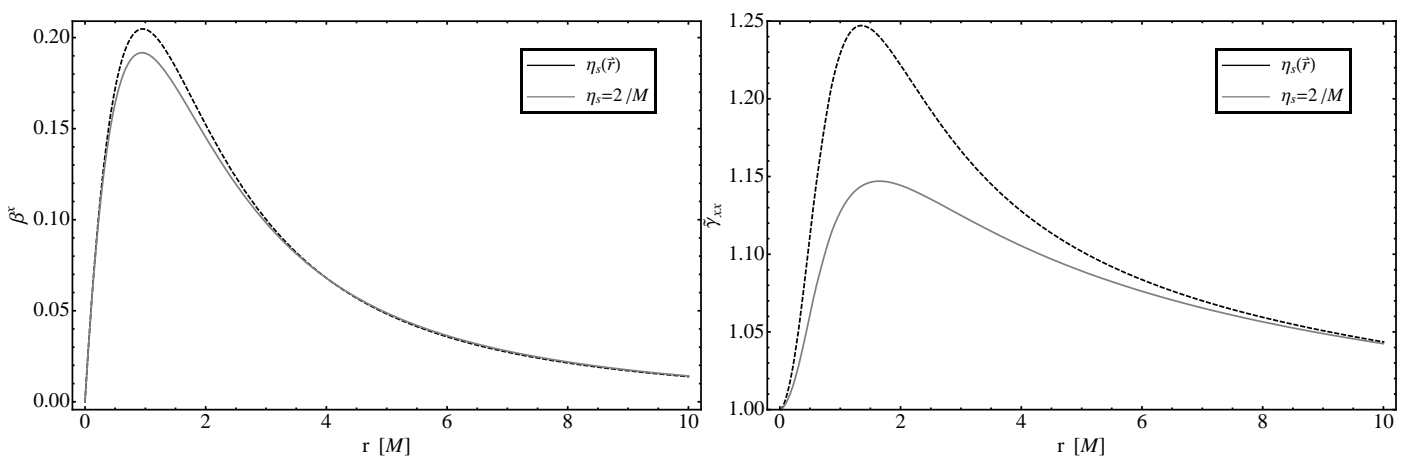

Figure 2. $x$-component of the shift vector (upper panel) and $x x$-component of the conformal 3-metric (lower panel) of a single, non-spinning puncture at time $t=100 \mathrm{M}$, where the simulations have reached a stationary state. The dashed black curves use dynamical damping, Eq. (4), the gray ones use $\eta_{s}=2.0 / M$ in the shift condition Eq. (2).

and therefore fulfills the limit $r \rightarrow \infty$ of (14) even though the outer boundary is situated only at $130 \mathrm{M}$.

Using a modified shift condition, the shift itself will, of course, change. We compare the $x$-component of the shift vector for using $\eta_{s}=2.0 / M$ and $\eta_{s}(\vec{r})$ in Fig. 2. A change in the shift implies a change of the coordinates and therefore, coordinate dependent quantities will change, too. As an example, the $x x$-component of the conformal 3-metric, $\tilde{\gamma}_{x x}$, is compared for $\eta_{s}=2 / M$ and $\eta_{s}(\vec{r})$ in the lower panel of Fig. 2. The comparisons are made at time $t=100 \mathrm{M}$, when the simulations have reached a stationary state.

The changes in the shift should only affect the coordinates and coordinate independent quantities should not change. This can be examined by looking at a scalar as a function of another scalar, e.g. the lapse $\alpha$ as a function of extrinsic curvature $K$, $\alpha=\alpha(K)$. Both scalars should see the same coordinate drifts and therefore, no changes 


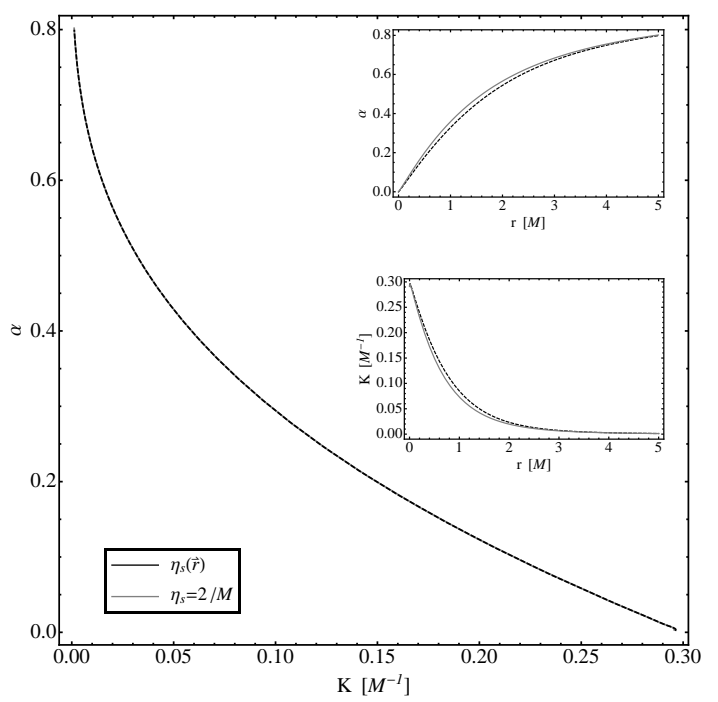

Figure 3. The lapse function $\alpha$ as a function of extrinsic curvature $K$ for a single, non-spinning and non-moving puncture after a time $t=50 \mathrm{M}$. We compare using $\eta_{s}(\vec{r})$ (black, dashed line) and $\eta_{s}=2.0 / M$ (gay line). The two curves lie perfectly on top of each other and are therefore indistinguishable. The insets show lapse (upper panel) and extrinsic curvature (lower panel) as functions of distance from the puncture.

are expected in $\alpha(K)$. Figure 3 confirms this expectation. The two curves $\alpha(K)$ for $\eta_{s}=2.0 / M$ and $\eta_{s}(\vec{r})$ are lying perfectly on top of each other. We therefore believe that using the dynamical damping introduces only coordinate changes in our puncture simulations.

\subsection{Black hole binary with equal masses}

While Eq. (4) has been introduced in order to allow for numerical simulations of two black holes with highly different masses, we first apply it to equal mass simulations in order to perform several consistency checks.

The (first order) coordinate independent quantity to look at in binary simulations is the Newman-Penrose scalar $\Psi_{4}$. We use $\Psi_{4}$ for the extraction of gravitational waves (see [7] for details of the wave extraction algorithm), decomposed into modes using spin-weighted spherical harmonics $Y_{l m}^{-2}$. Since $\Psi_{4}$ is only first-order gauge invariant and we furthermore extract waves at a finite, fixed coordinate radius, it is a priori an open question how much the changes in the shift affect the wave forms.

As the most dominant mode of $\Psi_{4}$ in an equal mass simulation is the $l=|m|=2$ mode, its real part multiplied by the extraction radius ( $r_{\mathrm{ex}}=90 \mathrm{M}$ in this case) is displayed in Fig. 4. We look at amplitude and phase of this mode in Figs. 5and 6] The initial separation was chosen to be $D=7 \mathrm{M}$. The black holes complete about 3 orbits. Three different resolutions are used corresponding to the three different colors in Figs. 4, 5and 6. We use the number of grid points in the inner boxes (centered around the black holes) to denote the different resolutions. The grid configurations, in the terminology of [7], are $\phi[5 \times 56: 5 \times 112: 6], \phi[5 \times 64: 5 \times 128: 6]$, and $\phi[5 \times 72: 5 \times 144: 6]$ 


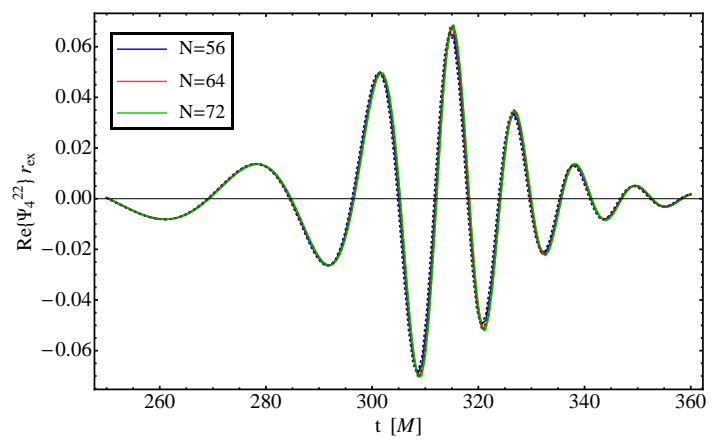

Figure 4. Real part of the 22-mode of $\Psi_{4}$ times extraction radius $r_{\text {ex }}$ for an equal mass binary with initial separation $D=7 M$ using $\eta_{s}=2.0 / M$ (solid lines) and $\eta_{s}(\vec{r})$ following Eq. (4) (dashed lines) in three different resolutions (blue, red, green lines) according to the grid configurations described in the text.

which corresponds to resolutions on the finest grids of $3 M / 112,3 M / 128$ and $M / 48$, respectively. These are the grid configurations used in [20].

In Fig. 5, we compare the amplitude $A_{22}$ in the standard gauge, $\eta_{s}=2.0 / M$, displayed as solid lines, to the new one, Eq. (44), plotted as dashed lines. We find that the differences between standard and new gauge for a given grid resolution are much smaller than differences due to using different resolutions. This strengthens the belief that we only introduced coordinate changes to the system when using Eq. (44). The maximum relative deviation between the amplitudes $A_{22}$ of old and new gauge amounts to about $3 \%$ for the lowest resolution $(N=56)$ and decreases with increasing resolution, which can be seen in the inset of Fig. 5. For the phase, the absolute differences are not visible by eye and therefore, we only plot the relative deviations between $\phi_{22}$ in the standard and new gauge for the three resolutions. For the lowest resolution $(N=56)$, the maximum deviation is only $0.35 \%$. As for the amplitude, this deviation decreases with increases in resolution. This shows how the differences in the waveforms disappear with increasing resolution.

The fact that there actually are differences visible in the waves, though very small ones, is not surprising when considering the way we extract gravitational waves. We fix a certain extraction radius and compute the Newman-Penrose scalar on a sphere of this radius. The radius itself is coordinate dependent and we are comparing $\Psi_{4}$ extracted at slightly different radii in the standard and new gauges. In future work we plan to compare wave forms extrapolated in radius to infinity, although it is worth noting how small the deviations are without additional processing.

\subsection{Black hole binary with mass ratio 4:1}

After having examined the influence of using a dynamical damping coefficient $\eta_{s}(\vec{r})$ for an equal mass binary, the next step is to look at its behavior for unequal masses. The following results are obtained from a simulation of two black holes with mass ratio $q=4$ and initial separation $D=7 M$. We used the grid configurations $\phi[5 \times N: 7 \times 2 N: 6]$ 


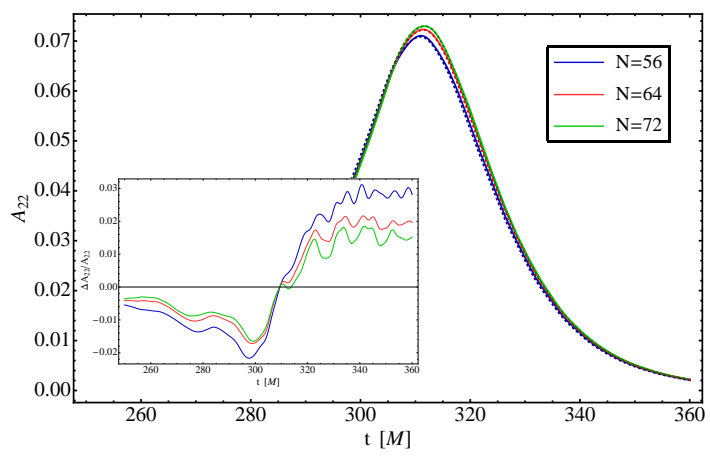

Figure 5. Amplitude of the 22-mode of $\Psi_{4}$ for the same binary as in Fig. (4) using $\eta_{s}=2.0 / M$ (solid lines) and $\eta_{s}(\vec{r})$ (dashed lines) in three different resolutions (blue, red, green lines) according to the grid configurations described in the text. The inset shows the relative deviation $\Delta A_{22} / A_{22}$ between the amplitude in the standard and in the new gauge, again for the three different resolutions.

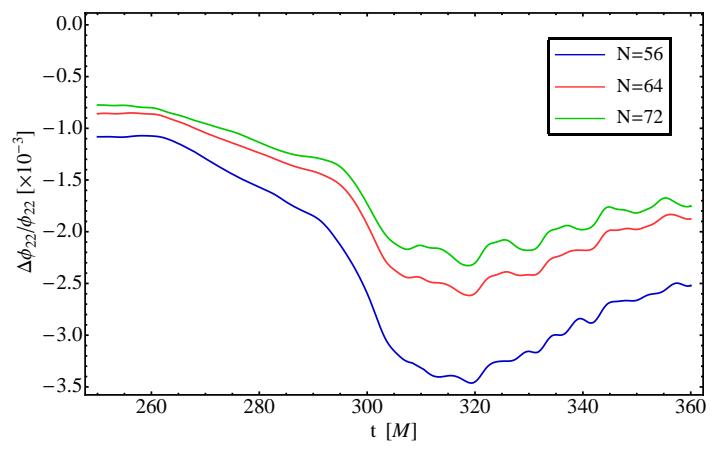

Figure 6. Relative phase difference of the 22-mode of $\Psi_{4}$ for the same binary as in Fig. (4) using $\eta_{s}=2 / M$ and $\eta_{s}(\vec{r})$. Compared are three different resolutions (blue, red and green lines) according to the grid configurations described in the text.

with $N=72,80$ which have also been used in 24] for mass ratio 4:1. An interesting question in this context is how Eq. (4) behaves for a simulation with two punctures with different masses. The analytical behavior (14) was deduced for a single, nonmoving, stationary puncture but now we are using it for two moving punctures, which are during most of the simulations far from having reached a stationary state globally, but approximately stationary locally at the punctures.

Figure 7 illustrates the distribution of $\eta_{s}(\vec{r})$ between the two punctures. For convenience, the conformal factor $\phi=\ln \psi$ is also plotted in order to indicate the positions of the punctures via its maxima (the divergences are not resolved). The snapshot is taken at a time during the simulation when the punctures are still well separated. Similar to the simulations of a single puncture, according to (14) we expect to find $\eta_{s}(\vec{r}) \simeq 2$ near the puncture with mass $M_{1}=0.5$ and $\eta_{s}(\vec{r}) \simeq 0.5$ in the vicinity of the second puncture with $M_{2}=2.0$. Near the outer boundary, $\eta_{s}$ is supposed to take the value $1.31 /\left(M_{1}+M_{2}\right)=0.52$. Here the $M_{i}$ are chosen to be dimensionless, so $\eta_{s}(\vec{r})$ is dimensionless as well. Figure 7 confirms that we do obtain the expected values, although they are not reached exactly. The latter is not a problem as simulations work 


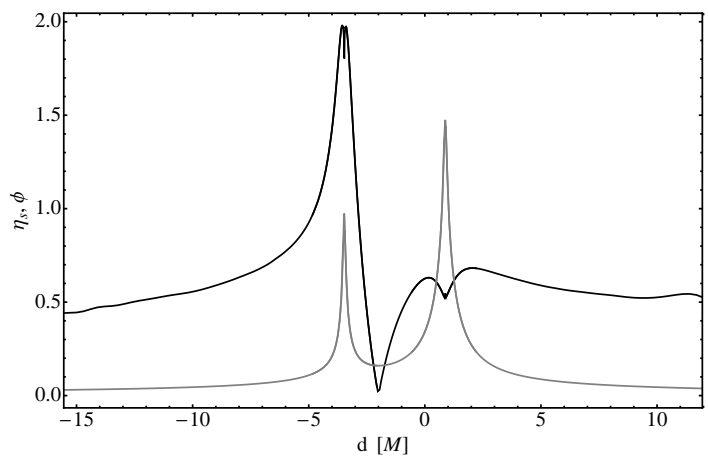

Figure 7. $\eta_{s}(\vec{r})$ (black line) for two punctures of bare masses $M_{1}=0.5$ and $M_{2}=2.0$ and the conformal factor $\phi$ (gray line) whose maxima show the current positions of the punctures. In this plot the $M_{i}$ are dimensionless, so $\eta_{s}(\vec{r})$ is dimensionless as well. The snapshot is taken right after the beginning of the $q=4$ simulation with initial separation $D=5 \mathrm{M}$ described in the text. The smaller black hole is at position $d=-3.6 \mathrm{M}$, the larger one at position $d=1.2 \mathrm{M}$.

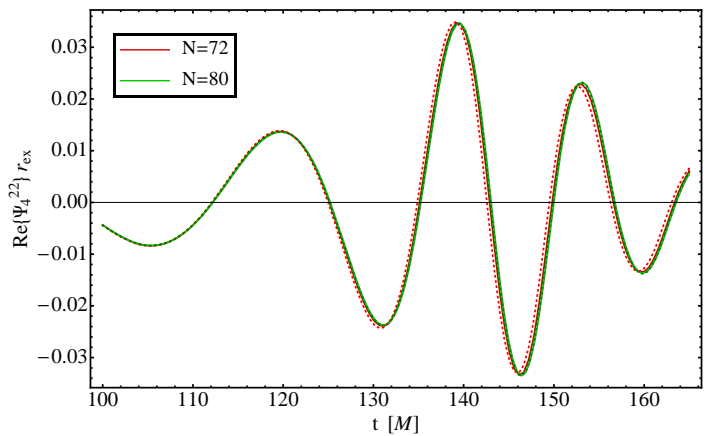

Figure 8. Real part of the 22-mode of $\Psi_{4}$ multiplied by the extraction radius $r_{\mathrm{ex}}$ for $q=4$ and initial separation $D=5 M$ runs. Compared are results for employing $\eta_{s}=2.0 / M$ (solid lines) and $\eta_{s}(\vec{r})$ (dashed lines) in two different resolutions (red and green lines) according to the grid configurations described in the text.

nicely as long as $\eta_{s}(\vec{r})$ is in the right range for each black hole. For this reason, Eq. (4) also seems to work rather nicely for two punctures with unequal masses.

As we did in the equal mass case in section 3.2 , we compare the 22-mode of $\Psi_{4}$ in the new gauge with the standard gauge. To see how small the differences using $\eta_{s}$ or $\eta_{s}(\vec{r})$ are, we plot its real part using two different resolutions which correspond to the two different colors in Fig. 8. Figure 9 shows the amplitude for the two different resolutions. The inset gives the relative differences between amplitudes in the standard and new gauge. The maximum relative deviation appears for the lower resolution and amounts to about $3 \%$. The high resolution gives $0.5 \%$ relative difference. The phases in standard and new gauge are compared in Fig. 10. Again, we show only the relative deviations as the absolute ones are too small to be seen. We find relative differences of up to $0.4 \%$ for the lower resolution and only $0.1 \%$ for the high one. This confirms that we are changing only the coordinates, as we found before in Sections 3.1 and 3.2 .

While the invariance of the waveforms is the most important feature of the new 


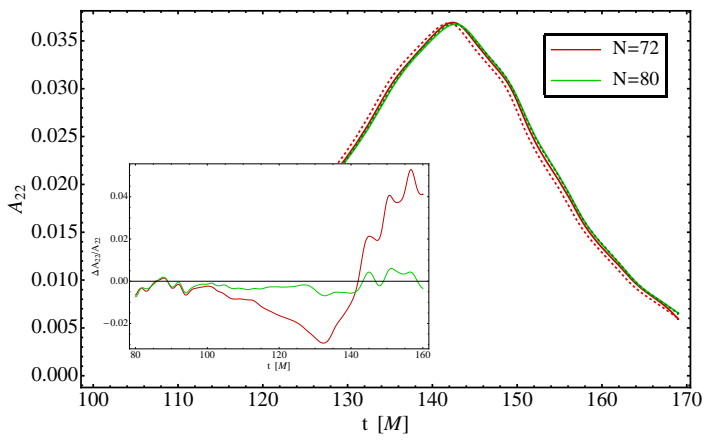

Figure 9. Amplitude of the 22-mode of $\Psi_{4}$ for the same runs as in Fig. 8 . We compare results using $\eta_{s}=2.0 / M$ (solid lines) and $\eta_{s}(\vec{r})$ (dashed lines) in two different resolutions (red and green lines) according to the grid configurations described in the text. The inset shows the relative deviation $\Delta A_{22} / A_{22}$ between the amplitude in the standard and in the new gauge, again for the same two resolutions.

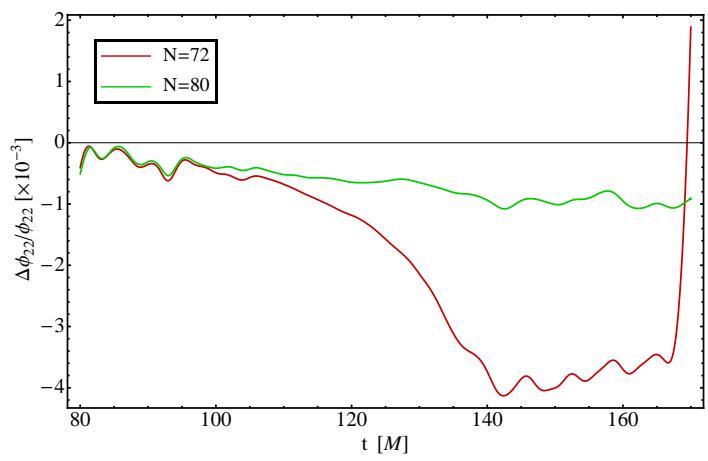

Figure 10. Relative phase difference of the 22-mode of $\Psi_{4}$ for the same run as in Fig. $8 \eta_{s}=2.0 / M$ and $\eta_{s}(\vec{r})$. Compared are two different resolutions according to the grid configurations described in the text.

gauge $\eta_{s}(\vec{r})$, it is illuminating to examine how the black holes are represented on the numerical grid. To this end, the apparent horizons $(\mathrm{AH})$ are computed for both gauges in Fig. 11. We show the result in the $(x, y)$-plane, in which the orbital plane lies. For clarity, the slices through the apparent horizons are only shown at 4 different times. In the beginning of the simulations, the AH pertaining to the same black hole are lying on top of each other. With time, they separate as the coordinates become more and more different in the two simulations. Two observations can be made. First, the ratio between the coordinate area of the $\mathrm{AH}$ of the larger black hole and the one of the smaller black hole is larger in the simulation using $\eta_{s}=2.0 / M$. This means the black holes are represented more equally on the grid in the simulation using Eq. (4). This fact can be seen even more clearly in Fig. 12 where we plot the coordinate area of the apparent horizons comparing the standard gauge (red lines) and the new one (black lines). While the coordinate sizes of the smaller black hole (dashed lines) are nearly equal in both gauges, the sizes of the larger black hole (solid lines) differ by roughly $2 M^{2}$. Second, the shape of the horizon of the smaller black hole is more and more distorted in the $\eta_{s}=2.0 / M$-simulation when the black holes come closer together. This deformation is 

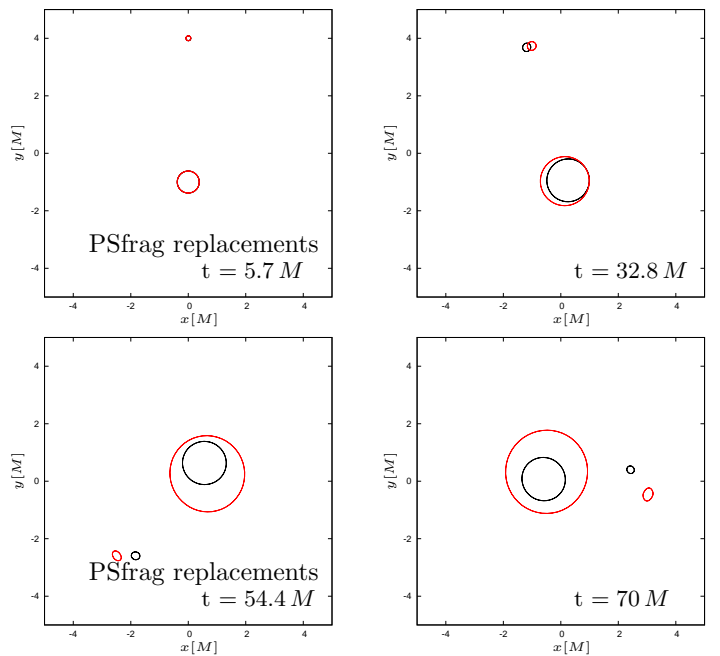

Figure 11. Comparison of apparent horizons in the orbital plane using $\eta_{s}=2.0 / \mathrm{M}$ (red lines) and $\eta_{s}(\vec{r})$ (black lines) at different times during the evolution for a $q=4$ run with initial separation $D=5 \mathrm{M}$.

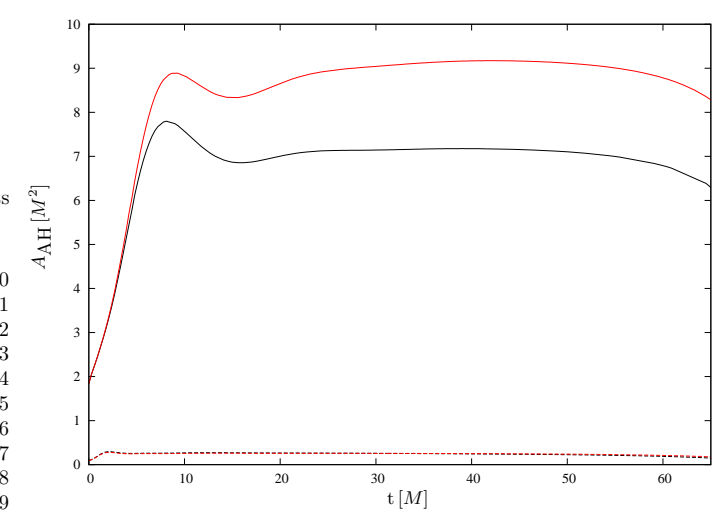

Figure 12. Comparison of the coordinate area of the apparent horizons using $\eta_{s}=2.0 / M$ (red lines) and $\eta_{s}(\vec{r})$ (black lines) over evolution time for a $q=4$ run with initial separation $D=5 M$ until shortly before a common apparent horizon appears. The dashed lines belong to the smaller black hole whereas the solid lines represent the larger black hole.

not visible in the new coordinates. The progressive stretching of the apparent horizon shape and therefore the distortion of the coordinates near the black holes can be a source of instabilities, e.g. [25]. Using Eq. (4) seems to be profitable in this regard.

\subsection{Behavior of $\eta_{s}(\vec{r})$ and influence on the shift vector}

Despite the encouraging results we have seen so far, there is a non-negligible concern using Eq. (44) in the gamma-driver condition (2). Although we do not determine the damping coefficient via a wave equation, we see wavy features in $\eta_{s}(\vec{r})$ traveling outwards. These distortions even leave remnants on the grid, especially when they pass through a refinement boundary. The form of $\eta_{s}(x)$ after different evolution times can 


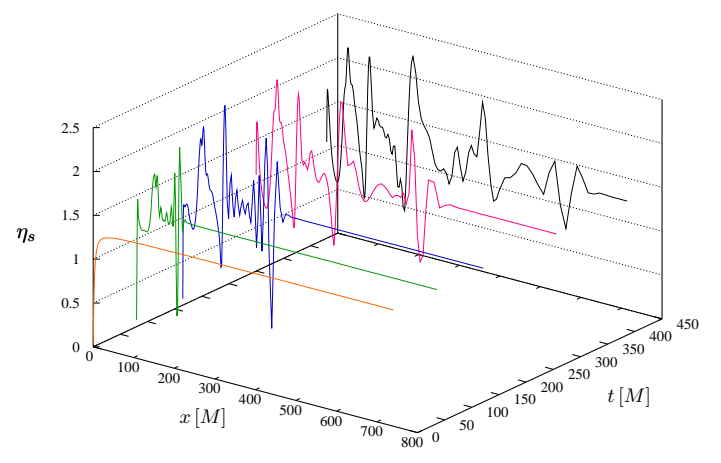

Figure 13. Form of $\eta_{s}(\vec{r})$ in $x$-direction at different times during an equal mass binary simulation. Noise travels outwards and leaves strong distortions on the grid.

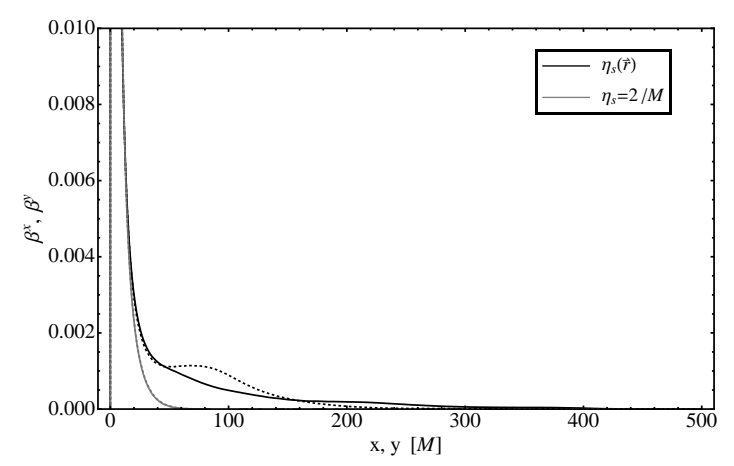

Figure 14. $x$-component of the shift vector in $x$-direction (solid curves) and $y$ component of the shift vector in $y$-direction (dashed curves) after the merger of two equal mass black holes at time $t=500 \mathrm{M}$ when using either the standard gauge $\eta_{s}=2.0 / M$ (gray lines) or the dynamical one, $\eta_{s}(\vec{r})$, (black lines).

be seen in Fig. 13 for the equal mass binary described in Sec. 3.2. The result is similar in the $q=4$ simulation and even in the Schwarzschild simulation, an outward traveling pulse is present, which however does not leave visible distortions on the grid and the relative amplitude of which decreases for higher mass. The effort we made before in order to achieve the correct value of $\eta_{s}(\vec{r})$ near the outer boundary seem to be canceled out by the disturbed shape we find now. As the peaks travel to a region of the grid where we have no punctures, we might take the point of view that the exact value of $\eta_{s}(\vec{r})$ and therefore the distortions are of no importance for our simulations. Indeed the oscillations do not translate to oscillations in the shift vector as one might think. In the shift, we find no gauge "waves" related to the ones in $\eta_{s}(\vec{r})$. Nevertheless, there is an unusual behavior. After merger, when going away from the punctures the shift does not fall off to zero as fast as it does when using $\eta_{s}=$ const. but keeps a shoulder (compare Fig. (14) which might lead to an unphysical and unwanted drift of the coordinate system. We are planning to investigate these issues in more detail in the future. 


\section{Discussion}

We presented a new approach to determine the coordinates in slices of spacetime for binary black hole simulations where we take the distribution of mass over the grid into account. We have shown that our approach of determining the damping parameter in the gamma-driver condition dynamically via Eq. (4) gives stable evolutions and does not significantly change the gravitational waves extracted from binary systems of equal or unequal masses. Furthermore, the use of Eq. (4) in an unequal mass simulation resulted in a more regular shape of the apparent horizon of the smaller black hole as the binary merges. The coordinate size of the apparent horizons became more uniform with the new damping coefficient which is a first step towards representing and resolving black holes with different masses equally and hence removing the large asymmetry which usually distorts the numerical grid in unequal mass simulations. We found gauge waves in our damping coefficient which might affect the stability in very long-term simulations and lead to coordinate drifts after the merger of the binary. We will address these issues in a future publication [26].

\section{Acknowledgments}

It is a pleasure to thank Jason Grigsby for discussions and for his valuable comments on this publication. We also thanks David Hilditch for discussions on the hyperbolicity of the BSSN system. This work was supported in part by DFG grant SFB/Transregio 7 "Gravitational Wave Astronomy" and the DLR (Deutsches Zentrum für Luft und Raumfahrt). Doreen Müller was additionally supported by the DFG Research Training Group 1523 "Quantum and Gravitational Fields". Computations were performed on the HLRB2 at LRZ Munich.

\section{References}

[1] J. Balakrishna, G. Daues, E. Seidel, W.-M. Suen, M. Tobias, and E. Wang. Coordinate conditions in three-dimensional numerical relativity. Class. Quantum Grav., 13:L135-142, 1996.

[2] Miguel Alcubierre and Bernd Brügmann. Simple excision of a black hole in $3+1$ numerical relativity. Phys. Rev. D, 63:104006, 2001.

[3] Miguel Alcubierre, Bernd Brügmann, Denis Pollney, Edward Seidel, and Ryoji Takahashi. Black hole excision for dynamic black holes. Phys. Rev. D, 64:061501(R), 2001.

[4] Miguel Alcubierre, Bernd Brügmann, Peter Diener, Michael Koppitz, Denis Pollney, Edward Seidel, and Ryoji Takahashi. Gauge conditions for long-term numerical black hole evolutions without excision. Phys. Rev. D, 67:084023, 2003.

[5] Manuela Campanelli, Carlos O. Lousto, Pedro Marronetti, and Yosef Zlochower. Accurate evolutions of orbiting black-hole binaries without excision. Phys. Rev. Lett., 96:111101, 2006.

[6] John G. Baker, Joan Centrella, Dae-Il Choi, Michael Koppitz, and James van Meter. Gravitational wave extraction from an inspiraling configuration of merging black holes. Phys. Rev. Lett., 96:111102, 2006.

[7] Bernd Brügmann, José A. González, Mark Hannam, Sascha Husa, Ulrich Sperhake, and Wolfgang Tichy. Calibration of moving puncture simulations. Phys. Rev. D, 77:024027, 2008. 
[8] Frank Herrmann, Deirdre Shoemaker, and Pablo Laguna. Unequal-mass binary black hole inspirals. 2006.

[9] José A. González, Ulrich Sperhake, and Bernd Brügmann. Black-hole binary simulations: the mass ratio 10:1. Phys. Rev., D79:124006, 2009.

[10] Miguel Alcubierre, Bernd Brügmann, Peter Diener, Frank Herrmann, Denis Pollney, Edward Seidel, and Ryoji Takahashi. Testing excision techniques for dynamical 3D black hole evolutions. 2004.

[11] Y. Zlochower, J. G. Baker, M. Campanelli, and C. O. Lousto. Accurate black hole evolutions by fourth-order numerical relativity. Phys. Rev. D, 72:024021, 2005. gr-qc/0505055.

[12] C. Bona, J. Massó, E. Seidel, and J. Stela. New Formalism for Numerical Relativity. Phys. Rev. Lett., 75:600-603, July 1995.

[13] Horst Beyer and Olivier Sarbach. On the well posedness of the Baumgarte-Shapiro- ShibataNakamura formulation of Einstein's field equations. Phys. Rev. D, 70:104004, 2004.

[14] Carsten Gundlach and Jose M. Martin-Garcia. Well-posedness of formulations of the Einstein equations with dynamical lapse and shift conditions. Phys. Rev. D, 74:024016, 2006.

[15] James R. van Meter, John G. Baker, Michael Koppitz, and Dae-Il Choi. How to move a black hole without excision: gauge conditions for the numerical evolution of a moving puncture. Phys. Rev. D, 73:124011, 2006.

[16] M. Shibata and T. Nakamura. Evolution of three-dimensional gravitational waves: Harmonic slicing case. Phys. Rev. D, 52:5428-5444, 1995.

[17] T. W. Baumgarte and S. L. Shapiro. On the Numerical integration of Einstein's field equations. Phys. Rev. D, 59:024007, 1998.

[18] Mark Hannam, Sascha Husa, Denis Pollney, Bernd Brügmann, and Niall Ó Murchadha. Geometry and regularity of moving punctures. Phys. Rev. Lett., 99:241102, 2007.

[19] Bernd Brügmann. Schwarzschild black hole as moving puncture in isotropic coordinates. Gen. Rel. Grav., 41:2131-2151, 2009.

[20] Sascha Husa, José A. González, Mark Hannam, Bernd Brügmann, and Ulrich Sperhake. Reducing phase error in long numerical binary black hole evolutions with sixth order finite differencing. Class. Quantum Grav., 25:105006, 2008.

[21] Marsha J. Berger and Joseph Oliger. Adaptive mesh refinement for hyperbolic partial differential equations. J. Comput. Phys., 53:484-512, 1984.

[22] Marcus Ansorg, Bernd Brügmann, and Wolfgang Tichy. A single-domain spectral method for black hole puncture data. Phys. Rev. D, 70:064011, 2004.

[23] Benny Walther, Bernd Brügmann, and Doreen Müller. Numerical black hole initial data with low eccentricity based on post-Newtonian orbital parameters. Phys. Rev., D79:124040, 2009.

[24] Thibault Damour, Alessandro Nagar, Mark Hannam, Sascha Husa, and Bernd Brügmann. Accurate effective-one-body waveforms of inspiralling and coalescing black-hole binaries. Phys. Rev., D78:044039, 2008.

[25] Bernd Brügmann, Wolfgang Tichy, and Nina Jansen. Numerical simulation of orbiting black holes. Phys. Rev. Lett., 92:211101, 2004.

[26] Doreen Müller, Jason Grigsby, and Bernd Brügmann. Dynamical damping for unequal mass black hole binary simulations. 2009. in preparation. 